\title{
Momentum distribution, $x$-ray scattering factor, and off-diagonal electron density generated by a bare Coulomb potential in the nonrelativistic limit of a large number of closed shells
}

\author{
I. A. Howard \\ Department of Chemistry (ALGC), Free University of Brussels (VUB), B-1050 Brussels, Belgium \\ N. H. March \\ Department of Physics, University of Antwerp, Groenenborgerlaan 171, B-2020 Antwerp, Belgium \\ and Oxford University, Oxford, England \\ L. M. Nieto* \\ Departamento de Física Teórica, Atómica y Óptica, Universidad de Valladolid, 47071 Valladolid, Spain
}

(Received 30 November 2007; published 11 March 2008)

\begin{abstract}
In earlier work, one of us displayed an exact Thomas-Fermi (TF) method for kinetic energy and other averages of the Compton profile for atomic closed shells in a bare Coulomb field. Here, we are also interested in the semiclassical TF-like method but now in relation to the momentum density itself in the limit of a large number of closed shells. Exact off-diagonal density matrices are also given explicitly, for both configuration and momentum spaces. Finally the x-ray scattering factor is discussed in relation to the Thomas-Fermi limit.
\end{abstract}

DOI: 10.1103/PhysRevA.77.032504

\section{INTRODUCTION}

The so-called $1 / Z$ expansion [1], a pillar of analytical atomic theory, has as its leading term the hydrogenic limit of the bare Coulomb potential $-Z e^{2} / r$. Therefore it remains of considerable interest to display compactly the properties of large numbers of closed shells generated by this potential. One such result is the spatial generalization [2] of Kato's theorem [3]. This generalization, which applies to an arbitrary number of closed shells reads [2]

$$
\frac{\partial n(r)}{\partial r}=-\frac{2 Z}{a_{0}} n_{s}(r), \quad a_{0}=\frac{\hbar^{2}}{m_{e} e^{2}},
$$

where $n(r)$ is the total electron density while $n_{s}(r)$ denotes the $s$-wave only $(\ell=0)$ component of $n(r)$. This relation (1) was briefly discussed in relation to the semiclassical limit in [2].

Defining the $\mathrm{x}$-ray scattering factor $f(k)$ in the customary way by

$$
f(k)=\int_{0}^{\infty} n(r) \frac{\sin k r}{k r} 4 \pi r^{2} d r,
$$

we see immediately that $f(0)=\int_{0}^{\infty} n(r) 4 \pi r^{2} d r$, which is the total number of electrons $N$ under consideration. For $\mathcal{N}$ closed shells in a bare Coulomb field we have, for singly occupied levels,

$$
N=\sum_{m=1}^{\mathcal{N}} m^{2}=\frac{\mathcal{N}(\mathcal{N}+1)(2 \mathcal{N}+1)}{6},
$$

\footnotetext{
*1uismi@metodos.fam.cie.uva.es
}

PACS number(s): 31.15.xg, 05.30.Fk, 71.10.Ca, 31.15.bt and for a small number of closed shells, $f_{\mathcal{N}}(k)$ has been discussed by Yusaf, Lawes, and March [4]. Below, our main concern will be with $f_{\mathcal{N}}(k)$ in the limit of large $\mathcal{N}$ and its relation to semiclassical Thomas-Fermi (TF) theory.

The third quantity, on which we shall focus particular attention, is the momentum density $\rho_{\mathcal{N}}(p)$, again for an arbitrary number of closed shells, but with some emphasis on the large $\mathcal{N}$ limit. This momentum distribution was given by Howard, March, and Van Doren [5] in terms of the polygamma function [see Eqs. (4) and (5) below]. Here, this exact result form $\rho_{\mathcal{N}}(p)$ will first be examined numerically in the large $\mathcal{N}$ limit, and then will be considered in relation to the TF semiclassical method.

Finally, we shall return to configuration space to obtain the off-diagonal density, or the Dirac density matrix $\gamma\left(\mathbf{r}, \mathbf{r}^{\prime}\right)$, in relation, as in Eq. (1), to the $s$-state only form $\gamma_{s}\left(\mathbf{r}, \mathbf{r}^{\prime}\right)$. A general relation was given, for example, by Theophilou and March [6]. This leads us naturally back to an equivalent $\mathbf{p}$ space result for the off-diagonal momentum density $\widetilde{\gamma}\left(\mathbf{p}, \mathbf{p}^{\prime}\right)$.

\section{MOMENTUM DENSITY $\rho_{\mathcal{N}}(p)$ IN THE LIMIT OF A LARGE NUMBER OF CLOSED SHELLS: EXACT RESULTS IN RELATION TO THE SEMICLASSICAL THOMAS-FERMI RESULT}

In Eq. (4.3) of [5], $\rho_{\mathcal{N}}(p)$ is given for a general number of closed shells in terms of the polygamma function $\psi^{(\mathcal{N})}(z)$, defined as the derivative of the gamma function [7] as

$$
\psi^{(\mathcal{N})}(z)=\frac{d^{\mathcal{N}+1} \ln \Gamma(z)}{d z^{\mathcal{N}+1}} .
$$

We shall quote here only the result as the number of closed shells tends to infinity given by Eq. (4.4) of [5] 


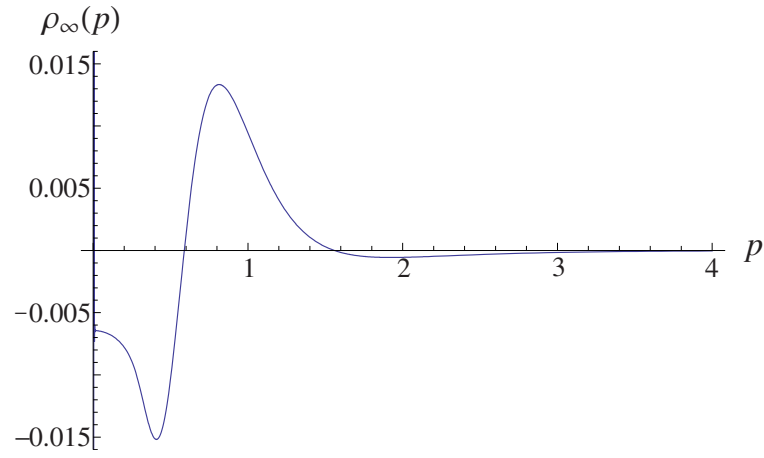

FIG. 1. (Color online) Plot of $\rho_{\infty}(p)$ versus $p$ for $p \geq 0$ when we remove the singular behavior at small $p$ shown in Eq. (6). All the quantities are in atomic units.

$$
\begin{aligned}
\rho_{\infty}(p)= & \frac{-Z^{2}}{6 \pi^{2} p^{9}}\left[-3 i p^{4}-2 i Z^{4} \pi^{4}+15 i \pi^{2} Z^{2} p^{2}\right. \\
& -30 i p^{2} \psi^{(1)}(i Z / p)+18 p Z^{3} \psi^{(2)}(i Z / p) \\
& +2 i Z^{4} \psi^{(3)}(i Z / p)-18 i p Z^{3} \pi^{3} \operatorname{coth}(\pi Z / p) \\
& +8 i Z^{4} \pi^{4} \operatorname{coth}^{2}(\pi Z / p)-15 i p^{2} Z^{2} \pi^{2} \operatorname{coth}^{2}(\pi Z / p) \\
& \left.+18 i p Z^{3} \pi^{3} \operatorname{coth}^{3}(\pi Z / p)-6 i Z^{4} \pi^{4} \operatorname{coth}^{4}(\pi Z / p)\right] .
\end{aligned}
$$

This exact quantal form will be used immediately below, for $Z=1$, to make contact with semiclassical theory. However, we note that an expansion of $\rho_{\infty}(p)$ to display the singular behavior as $p \rightarrow 0$ has the form

$$
\begin{aligned}
\rho_{\infty}(p)= & \frac{8}{3 \pi^{2}} \frac{1}{p^{6}}-\frac{4}{63 \pi^{2}}-\frac{4}{15 \pi^{2}} p^{2}-\frac{40}{33 \pi^{2}} p^{4}-\frac{5528}{819 \pi^{2}} p^{6} \\
& +\cdots
\end{aligned}
$$

To emphasize the nature of Eqs. (5) and (6), we show in Fig. 1 a plot of $\rho_{\infty}(p)$ versus $p$ for $p \geq 0$ when we remove the singular behavior at small $p$ shown in Eq. (6). Then, the resulting form has the rather simple behavior shown in Fig. 1.

\section{A. Comparison with the semiclassical Thomas-Fermi limit}

As discussed, for example, by Coulson and March [8], the TF semiclassical density $n_{T F}(r)$ is related to the maximum momentum $p(r)$ at position $r$ by

$$
n_{T F}(r)=\frac{p^{3}(r)}{6 \pi^{2}}
$$

The semiclassical nature of Eq. (7) is evident from the fact that given an appropriate electron density $n(r)$, one can extract a precise momentum $p(r)$ at position $r$. Also, it follows [8] that one can extract a momentum density from the electron density, as given in the following equation. This momentum density $\rho_{T F}(p)$ can be expressed via Eq. (7) as
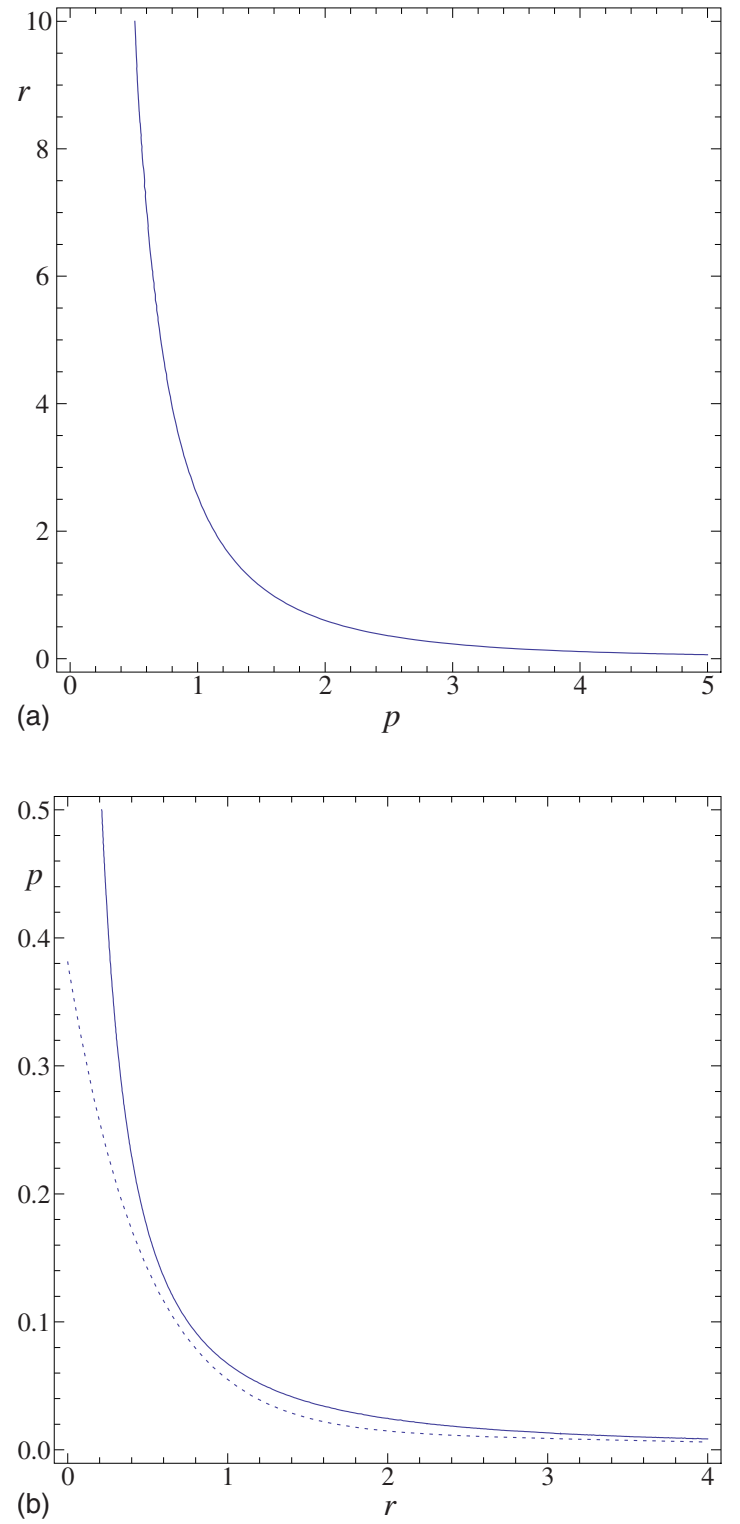

FIG. 2. (Color online) (a) Plot of semiclassical Thomas-Fermi quantity $r(p)$ in Eq. (8), but now with the left-hand side of this equation replaced by $\rho_{\infty}(p)$ calculated from Eq. (5) with $Z=1$. Part (b) uses part (a) for $r(p)$ to extract $p(r)$ to insert in the rhs of Eq. (7). This "refined semiclassical $\mathbf{r}$ space density" (solid line) is then compared here with the Heilmann-Lieb [9] form $n_{\infty}(r)$, again for $Z=1$ (dashed line). All the quantities are in atomic units.

$$
\rho_{T F}(p)=\frac{r^{3}(p)}{6 \pi^{2}}
$$

To test the utility of the semiclassical TF equations (7) and (8) in the favorable limit as the number of closed shells tends to infinity, we show $r(p)$ obtained from the exact momentum density (5) (see caption of Fig. 2) in Fig. 2(a) and in Fig. 2(b) the related quantity $p^{3}(r) /\left(6 \pi^{2}\right)$ entering Eq. (7). For comparison, the Heilmann-Lieb [9] density $n_{\infty}(r)$ is shown for the case $Z=1$. The accord in Fig. 2(b) is satisfactory, except near the origin where the semiclassical approximation breaks down. 


\section{B. Off-diagonal density in p space for the $m$ th closed shell}

May [10] generalized the momentum density of Fock [11] for the $m$ th closed shell away from the diagonal. May's result is essentially for the off-diagonal density matrix $\widetilde{\gamma}_{m}\left(\mathbf{p}, \mathbf{p}^{\prime}\right)$ for the $m$ th closed shell which he obtains as

$$
\tilde{\gamma}_{m}\left(\mathbf{p}, \mathbf{p}^{\prime}\right)=\frac{2^{6} \pi^{2} m^{4}}{\lambda^{3} \sqrt{2 \pi}} \frac{T_{m-1}^{1 / 2}\left(\cos \theta \cos \theta^{\prime}+\sin \theta \sin \theta^{\prime} \cos \phi\right)}{\left(z^{2}+1\right)^{2}\left(z^{\prime 2}+1\right)^{2}},
$$

where $\phi$ is the angle between $\mathbf{p}$ and $\mathbf{p}^{\prime}, z=m p / \lambda, z^{\prime}$ $=m p^{\prime} / \lambda$, and $\lambda=Z^{-1}$,

$$
\cos \theta=\frac{z^{2}-1}{z^{2}+1}, \quad \cos \theta^{\prime}=\frac{z^{\prime 2}-1}{z^{\prime 2}+1},
$$

and consequently

$$
\sin \theta=\frac{2 z}{z^{2}+1}, \quad \sin \theta^{\prime}=\frac{2 z^{\prime}}{z^{\prime 2}+1} .
$$

$T_{n}^{1 / 2}(x)$ are Chebyshev polynomials and May gives

$$
T_{n-1}^{1 / 2}(1)=\sqrt{2 / \pi} n,
$$

leading to

$$
\tilde{\gamma}_{m}(\mathbf{p}, \mathbf{p})=\rho_{m}(p)=\frac{2^{6} \pi m^{5}}{\lambda^{3}\left(z^{2}+1\right)^{4}},
$$

which recovers the result of Fock for the momentum density of the $m$ th closed shell. We shall return, albeit briefly, to the off-diagonal form of Eq. (13) at the end of Sec. III below.

\section{ELECTRON DENSITY AND ITS OFF-DIAGONAL GENERALIZATION}

The electron density $n_{m}(r)$ of the $m$ th closed shell satisfies the third-order nonlinear differential equation [2]

$$
r n_{m}^{\prime \prime \prime}(r)-\frac{r}{2}\left(\frac{n_{m}^{\prime \prime}(r)}{n_{m}^{\prime}(r)}\right)^{2} n_{m}^{\prime}(r)+2 n_{m}^{\prime \prime}(r)+\left(4 Z-\frac{2 Z^{2} r}{m^{2}}\right) n_{m}^{\prime}(r)=0 .
$$

The order can be reduced as Cooper subsequently showed using the methods of supersymmetric quantum mechanics for the radial Coulomb problem [12]. The reduced order differential equation reads

$$
\begin{aligned}
& n_{m}^{\prime \prime}(r)+\frac{r}{4}\left(\frac{n_{m}^{\prime \prime}(r)}{n_{m}^{\prime}(r)}\right)^{2} n_{m}^{\prime}(r)-\left(\frac{Z^{2} r}{m^{2}}-\frac{1}{r}-2 Z\right) n_{m}^{\prime}(r)+\frac{2 Z}{r} n_{m}(r) \\
& \quad=0 .
\end{aligned}
$$

Eliminating the nonlinear term from Eq. (14) using Eq. (15) yields then the third-order linear homogeneous differential equation

$$
r^{2} n_{m}^{\prime \prime \prime}(r)+4 r n_{m}^{\prime \prime}(r)+\left(2+8 Z r-\frac{4 Z^{2} r^{2}}{m^{2}}\right) n_{m}^{\prime}(r)+4 Z n_{m}(r)=0 .
$$
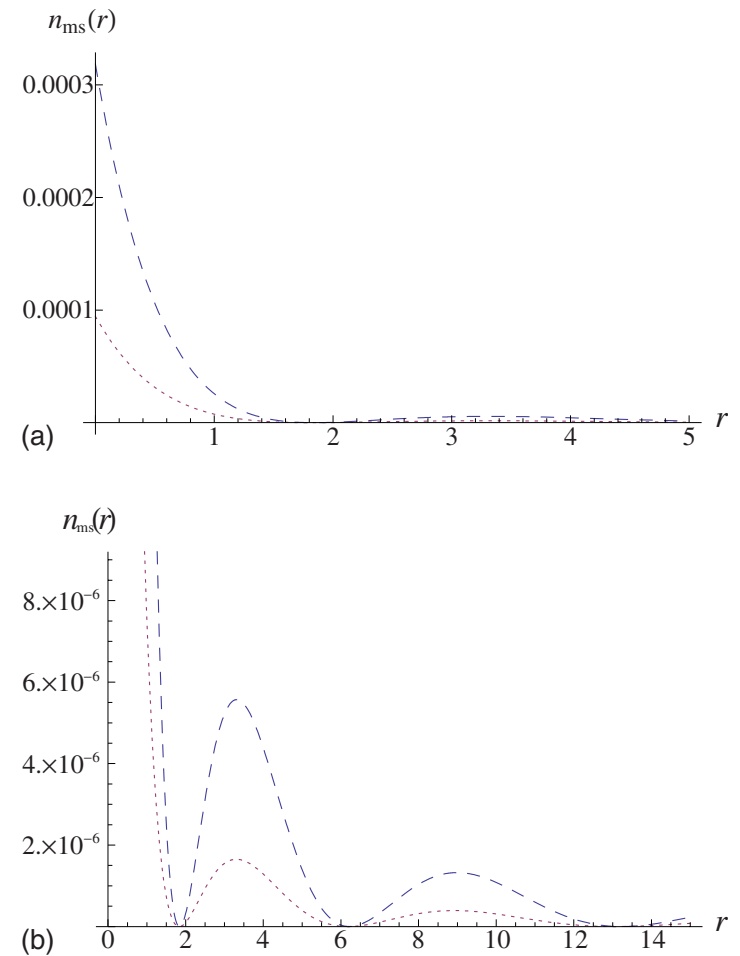

FIG. 3. (Color online) Density $n_{m s}(r)$ given in Eq. (18) for $m$ $=10$ (dashed line) and $m=15$ (dotted line). The full curves are presented in (a) for $r \in[0,5]$ and in (b) detail is shown for $r \in[0,15]$. All the quantities are in atomic units.

We have verified using MATHEMATICA that the physical solution of Eq. (16), which is therefore finite at $r=0$ and tends to zero at infinity, is given explicitly, for $Z=1$ and in atomic units by

$$
\begin{aligned}
n_{m}(r)= & \frac{1}{\pi m^{4}} e^{-2 r / m}\left\{m\left[L_{m-1}^{(1)}\left(\frac{2 r}{m}\right)\right]^{2}+m\left[L_{m-2}^{(1)}\left(\frac{2 r}{m}\right)\right]^{2}\right. \\
& \left.+\left(\frac{2 r}{m}-2 m\right) L_{m-1}^{(1)}\left(\frac{2 r}{m}\right) L_{m-2}^{(1)}\left(\frac{2 r}{m}\right)\right\}
\end{aligned}
$$

where $L_{n}^{(\mu)}(x)$ are generalized Laguerre polynomials. The $s$ component of the total density $n_{m}(r)$ in Eq. (17) is easily obtained as

$$
n_{m s}(r)=\frac{m^{-5}}{\pi} e^{-2 r / m}\left[L_{m-1}^{(1)}\left(\frac{2 r}{m}\right)\right]^{2}
$$

and then it is readily shown that the first term on the righthand side (rhs) of Eq. (17) is simply $m^{2} n_{m s}(r)$, which contains the entire normalization condition

$$
\int_{0}^{\infty} n_{m}(r) 4 \pi r^{2} d r=m^{2} \int_{0}^{\infty} n_{m s}(r) 4 \pi r^{2} d r .
$$

The $s$-state density $n_{m s}(r)$ given in Eq. (18), which is indeed the square of the $s$-component radial wave function of principal quantum number $m$, is plotted in Fig. 3 for two values of $m$ greater than or equal to 10 . 


\section{A. $r$ space off-diagonal density}

The $\mathbf{r}$ space density matrix $\gamma_{m s}\left(r, r^{\prime}\right)$ was used by Theophilou and March [6] to construct the complete $\mathbf{r}$ space density matrix $\gamma_{m}\left(\mathbf{r}, \mathbf{r}^{\prime}\right)$ :

$$
\gamma_{m}\left(\mathbf{r}, \mathbf{r}^{\prime}\right)=-(x-y)^{-1}\left(\frac{\partial}{\partial x}-\frac{\partial}{\partial y}\right)\left[x y \gamma_{m s}\left(\frac{x}{2}, \frac{y}{2}\right)\right]
$$

where (see, e.g., [13])

$$
x=r+r^{\prime}+\left|\mathbf{r}-\mathbf{r}^{\prime}\right|, \quad y=r+r^{\prime}-\left|\mathbf{r}-\mathbf{r}^{\prime}\right| .
$$

Whereas Eq. (1) allows $n_{s}(r)$ to be obtained directly from the total density $n(r)$ [see, e.g., Eqs. (17) and (18)] the result (20) yields the total density matrix $\gamma_{m}$ by differentiation of $\gamma_{m s}$. From Eq. (18) for $n_{m s}(r)$, it follows that the off-diagonal generalization to $\gamma_{m s}\left(\mathbf{r}, \mathbf{r}^{\prime}\right)$ is readily verified to be

$$
\gamma_{m s}\left(r, r^{\prime}\right)=\frac{m^{-5}}{\pi} e^{-r / m} L_{m-1}^{(1)}\left(\frac{2 r}{m}\right) e^{-r^{\prime} / m} L_{m-1}^{(1)}\left(\frac{2 r^{\prime}}{m}\right) .
$$

Using Eq. (21), one immediately has $\gamma_{m s}(x / 2, y / 2)$ appearing on the rhs of Eq. (20). Taking the derivatives shown, one finds

$$
\begin{aligned}
\gamma_{m}\left(\mathbf{r}, \mathbf{r}^{\prime}\right)= & \frac{e^{-(x+y) / 2 m}}{m^{6} \pi(x-y)}\left\{-x y L_{m-2}^{(2)}\left(\frac{y}{m}\right) L_{m-1}^{(1)}\left(\frac{x}{m}\right)\right. \\
& \left.\times\left[x y L_{m-2}^{(2)}\left(\frac{x}{m}\right)+m(x-y) L_{m-1}^{(1)}\left(\frac{x}{m}\right)\right] L_{m-1}^{(1)}\left(\frac{y}{m}\right)\right\} .
\end{aligned}
$$

Thus it is clear from Eqs. (20) and (21) that the $s$-state density matrix for the $m$ th closed shell determines the total density matrix $\gamma_{m}\left(\mathbf{r}, \mathbf{r}^{\prime}\right)$ for that shell. Of course, it will be a considerable simplification if an analogous momentum space treatment could construct the total density matrix $\widetilde{\gamma}_{m}\left(\mathbf{p}, \mathbf{p}^{\prime}\right)$ from the explicit $s$-wave form in Eq. (31) below. A step toward this objective is to note that, apart from a normalization constant, the $s$-wave density matrices are related by

$$
\tilde{\gamma}_{m s}\left(p, p^{\prime}\right)=\int \gamma_{m s}\left(r, r^{\prime}\right) \exp \left(i\left[\mathbf{p} \cdot \mathbf{r}-\mathbf{p}^{\prime} \cdot \mathbf{r}^{\prime}\right]\right) d \mathbf{r} d \mathbf{r}^{\prime}
$$

Then, for the limited off-diagonal case of $\tilde{\gamma}_{m s}\left(p, p^{\prime}\right)$ corresponding to $p^{\prime}=0$ we find

$$
\widetilde{\gamma}_{m s}\left(p, p^{\prime}=0\right)=\int \gamma_{m s}\left(r, r^{\prime}\right) \exp (i \mathbf{p} \cdot \mathbf{r}) d \mathbf{r} d \mathbf{r}^{\prime},
$$

and making use of the explicit form (22) we find (see also Appendix A)

$$
\widetilde{\gamma}_{m s}\left(p, p^{\prime}=0\right)=8(-1)^{m+1} \int d \mathbf{r} e^{i \mathbf{p} \cdot \mathbf{r}} e^{-r / m} L_{m-1}^{(1)}(2 r / m),
$$

which prompts us therefore to return briefly to the complete momentum density matrix $\tilde{\gamma}_{m}\left(\mathbf{p}, \mathbf{p}^{\prime}\right)$ for the $m$ th closed shell.

\section{B. $\widetilde{\gamma}_{m}\left(\mathbf{p}, \mathbf{p}^{\prime}\right)$ revisited}

The work of Holstein [14] appears to simplify the expression of May [10] discussed above. In particular, for the $n$th closed shell Holstein's Eq. (29) gives, with $\psi$ denoting the momentum eigenfunction,

$$
\begin{aligned}
\sum_{\ell, m} \psi_{n \ell m}(\mathbf{p}) \psi_{n \ell m}^{*}\left(\mathbf{p}^{\prime}\right)= & \frac{1}{k_{n}^{2}+p^{2}} \frac{1}{k_{n}^{2}+p^{\prime 2}} \frac{m_{e} \alpha^{2}}{n^{3}} \frac{1}{(n-1) !} \\
& \times 16 \pi m_{e} \alpha^{2} k_{n}\left(2 m_{e}\right)^{2} \\
& \times\left.\frac{d^{n-1}}{d x^{n-1}} \frac{1}{L\left(\mathbf{p}, \mathbf{p}^{\prime}, x\right)}\right|_{x=0},
\end{aligned}
$$

where

$$
k_{n}^{2}=\frac{m_{e}^{2} \alpha^{2}}{2 n^{2}}
$$

Then Holstein's Eq. (33) relates the derivative in Eq. (27) to a Gegenbauer polynomial $C_{n-1}^{(1)}(t)$ by

$$
\left.\frac{d^{n-1}}{d x^{n-1}} \frac{1}{L\left(\mathbf{p}, \mathbf{p}^{\prime}, x\right)}\right|_{x=0}=\frac{(n-1) !}{\left(k_{n}^{2}+p^{2}\right)\left(k_{n}^{2}+p^{\prime 2}\right)} C_{n-1}^{(1)}(t),
$$

where finally, $t$ is given by his Eq. (34) which has misprints in the first term on the rhs. However, his second term on the rhs of Eq. (34) is correct and involves importantly $\cos \phi$, where $\phi$ is the angle between $\mathbf{p}$ and $\mathbf{p}^{\prime}$.

To conclude this section, let us return briefly to the $\mathbf{r}$ space result in Eq. (1). This relates the total density $n(r)$ to its $s$-wave $(\ell=0)$ component; but for a particular closed shell with principal quantum number $n$ and $\ell=m=0$, one has for the (singly occupied) $s$-state the result

$$
\tilde{\gamma}_{n s}\left(p, p^{\prime}\right)=\psi_{n 00}(p) \psi_{n 00}\left(p^{\prime}\right) .
$$

It then follows from Eq. (36) of Holstein (after misprints are corrected) that

$$
\begin{aligned}
\tilde{\gamma}_{n s}\left(p, p^{\prime}\right)= & 32 \pi^{2} m_{e} \alpha^{2} k_{n}^{2}\left(2 m_{e}\right)^{2} \frac{m_{e} \alpha}{n^{3}}\left(\frac{1}{k_{n}^{2}+p^{2}}\right)^{2}\left(\frac{1}{k_{n}^{2}+p^{\prime 2}}\right)^{2} \\
& \times \frac{1}{n} C_{n-1}^{(1)}\left(\frac{k_{n}^{2}-p^{2}}{k_{n}^{2}+p^{2}}\right) C_{n-1}^{(1)}\left(\frac{k_{n}^{2}-p^{\prime 2}}{k_{n}^{2}+p^{\prime 2}}\right)
\end{aligned}
$$

which is an off-diagonal generalization of the result (26) for $p^{\prime}=0$ (see also Appendix A).

\section{X-RAY SCATTERING FACTOR $f(k)$ FOR AN ARBITRARY NUMBER OF CLOSED SHELLS COMPARED WITH THE THOMAS- FERMI SEMICLASSICAL LIMIT}

As mentioned above, in [4] the x-ray scattering factor $f(k)$ defined in Eq. (2) was calculated for small numbers of closed shells. Here, because of our interest in the range of validity of the semiclassical TF result, we have taken the density $n_{\infty}(r)$ calculated by Heilmann and Lieb [9] for $Z=1$ and have obtained the $\mathrm{x}$-ray scattering factor $f_{\infty}(k)$. This is displayed in 


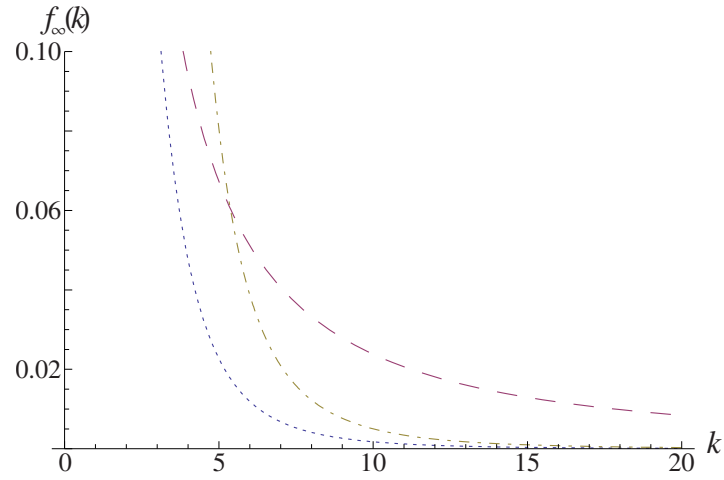

(a)

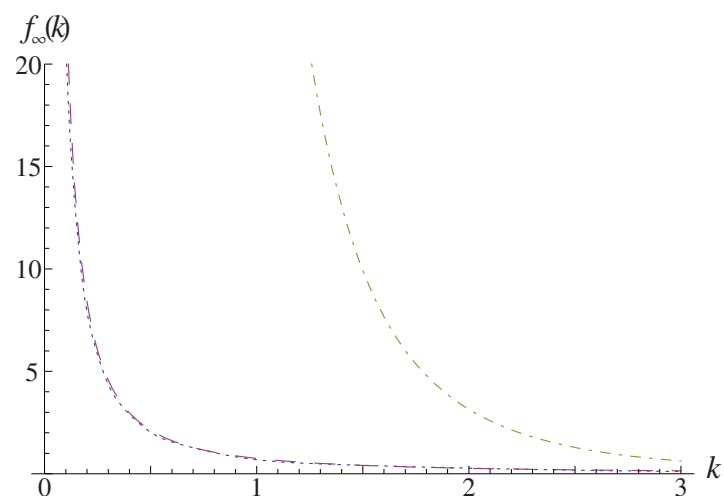

(b)

FIG. 4. (Color online) Plot of the x-ray scattering factor $f_{\infty}(k)$ (dotted lines) and of its asymptotic results for small $k$ given in Eq. (33) (dashed lines) and large $k$ given in Eq. (34) (dot-dashed lines); $k$ is given in atomic units. In (a) we can appreciate the good agreement between $f_{\infty}(k)$ and Eq. (34) for large values of $k$; in (b) we can see the good agreement between $f_{\infty}(k)$ and Eq. (33) for small values of $k$ [the two curves are almost indistinguishable, and very different from the dot-dashed line Eq. (34)]. All the quantities are in atomic units.

Fig. 4 for comparison with the TF limiting result. Analytic limits for small and large $k$ of $f_{\infty}(k)$ are recorded in Appendix $\mathrm{B}$ and are also displayed in Fig. 4.

We turn next to utilize again the linear differential equation (16) for the $m$ th closed shell density $n_{m}(r)$. From Eq. (2), the corresponding scattering factor $f_{m}(k)$ is readily obtained from Eq. (16) by multiplying throughout by $4 \pi r^{2}(\sin k r) /(k r)$, and then integrating, to obtain

$$
\begin{aligned}
f_{m}(k)= & -2 Z \int_{0}^{\infty}\left(\frac{Z r^{2}}{m^{2}}-2 r-\frac{1}{2 Z}\right) n_{m s}(r) \frac{\sin k r}{k r} 4 \pi r^{2} d r \\
& +2 \int_{0}^{\infty} r n_{m s}^{\prime}(r) 4 \pi r^{2} \frac{\sin k r}{k r} d r \\
& +\frac{1}{2} \int_{0}^{\infty} r^{2} n_{m s}^{\prime \prime}(r) 4 \pi r^{2} \frac{\sin k r}{k r} d r .
\end{aligned}
$$

Equation (32) therefore expresses the quantity $f_{m}(k)-f_{m s}(k)$, the total scattering factor for the $m$ th closed shell minus its $s$-state component, solely in terms of (derivatives of) the $s$-state $m$ th shell density $n_{m s}(r)$, and can be legitimately viewed therefore, even though less simple, as the analog of Eq. (1) for the x-ray scattering factor. Though $f_{m s}(k)$ has not, so far, been achieved in terms of known functions, we have used Eqs. (2) and (18) to show that it has the form $p_{2 m-2}\left((k m / 2)^{2}\right) /\left[1+(k m / 2)^{2}\right]^{2 m}$, where $p_{2 m-2}(x)$ is a polynomial of order $2 m-2$. Further detail is given in Appendix B.

Turning to the Fourier transform of $n_{\infty}(r)$, namely $f_{\infty}(k)$ via Eq. (2), one can use the approach set out in Appendix B to obtain asymptotic results for small and large $k$ as follows:

$$
f_{\infty}(k)=\frac{4}{3 \sqrt{\pi}} \frac{Z^{3 / 2}}{k^{3 / 2}}, \quad k \rightarrow 0
$$

and

$$
f_{\infty}(k)=\frac{16 \pi}{k^{4}} \frac{Z}{a_{0}} n_{\infty}(r=0), \quad k \rightarrow \infty,
$$

which, according to Heilmann and Lieb [9], can be expressed in terms of the Riemann zeta function because for $Z=1$ we have $n_{\infty}(r=0)=\zeta(3) / \pi \approx 0.383$ a.u. For the full treatment of $f_{\infty}(k)$ for arbitrary $k$, one can proceed numerically via Eqs. (B11)-(B14), but we shall not pursue further details.

\section{SUMMARY AND PROPOSED FUTURE DIRECTIONS}

The exact relation (5) has first been utilized to extract $r(p)$ from Eq. (8) as in Fig. 2(a) for $Z=1$. The result for $p(r)$ thus obtained has been inserted in Eq. (7) to obtain a "modified semiclassical density" for comparison with the exact Heilmann-Lieb form [9]. Except near the nucleus, the agreement is good in this admittedly favorable case for semiclassical theory. Turning to the electron density $n_{m}(r)$ for the $m$ th closed shell, the known nonlinear differential equations (14) and (15) have been shown to lead to the third-order linear homogeneous differential equation (16). The physical solution of this equation has been obtained and is given explicitly in terms of generalized Laguerre polynomials in Eq. (17). Importantly, we have also obtained the exact off-diagonal Dirac density matrix $\gamma_{m}\left(\mathbf{r}, \mathbf{r}^{\prime}\right)$, for the $m$ th closed shell, in Eq. (23), and have verified that this correctly reduces to $n_{m}(r)$ in Eq. (17) in the limit $\mathbf{r}^{\prime} \rightarrow \mathbf{r}$. The $\mathbf{r}$ space analog for the $s$-component of the momentum density matrix $\widetilde{\gamma}_{m s}\left(p, p^{\prime}\right)$ in Eq. (31) for the special case $p^{\prime}=0$ is given in Eq. (26).

Finally, analytic progress has proved possible on the x-ray scattering factor, both in its exact quantal form and in the semiclassical limit. The former is typified by Eqs. (32) and (B11), while the latter is given in closed form in Eq. (B6).

As to future directions, one can expect simplifications to subsequently emerge by relating the off-diagonal momentum density $\tilde{\gamma}\left(\mathbf{p}, \mathbf{p}^{\prime}\right)$ to its $s$-wave contribution $\tilde{\gamma}_{s}\left(p, p^{\prime}\right)$. This would then complement the $\mathbf{r}$ space relationship considered by Theophilou and March [6], quoted in Eqs. (20) and (21) above. These concern the role of the Runge-Lenz vector as an additional constant of motion in the hydrogenic limit considered throughout the present paper. 


\section{ACKNOWLEDGMENTS}

N.H.M. acknowledges that his contribution to the study started during a visit to the University of Antwerp, Belgium, which was partially supported by FWO-Vlaanderen Project No. G.0425.05. He also thanks Professor D. Van Dyck and Professor D. Lamoen for making possible continuing affiliation with the Physics Department, Antwerp. The contribution of N.H.M. was brought to fruition at the University of Valladolid. Professor J. A. Alonso and Professor M. Santander are thanked for their generous hospitality. This work has been partially supported by Spanish Ministerio de Educación y Ciencia (Project No. MTM2005-09183) and Junta de Castilla y León (Excellence Project No. VA013C05).

\section{APPENDIX A: LIMITED OFF-DIAGONAL INFORMATION IN MOMENTUM SPACE FOR $s$-STATE MATRIX$$
\widetilde{\gamma}_{m s}\left(\mathbf{p}, \mathbf{p}^{\prime}=\mathbf{0}\right)
$$

We show below that $\widetilde{\gamma}_{m s}\left(\mathbf{p}, \mathbf{p}^{\prime}=0\right)$ introduced in Eqs. (24) and (25) of the main text can be written in fact analytically as a quotient of two polynomials. Indeed, it is possible to readily accomplish the integration of the angular part in Eq. (25) to get

$$
\begin{aligned}
\tilde{\gamma}_{m s}\left(p, p^{\prime}=0\right)= & 32 \pi(-1)^{m+1} \int_{0}^{\infty} \exp (-r / m) \frac{\sin p r}{p r} \\
& \times L_{m-1}^{(1)}(2 r / m) r^{2} d r .
\end{aligned}
$$

Then, taking into account that

$$
L_{n}^{(\alpha)}(x)=\sum_{k=0}^{n}\left(\begin{array}{c}
n+\alpha \\
n-k
\end{array}\right) \frac{(-x)^{k}}{k !}
$$

with $\alpha=1, n=m-1$, and $x=(2 r / m)=2 y$, we get

$$
\begin{aligned}
\tilde{\gamma}_{m s}\left(p, p^{\prime}=0\right)= & 32 \pi(-1)^{m+1} \frac{m^{2}}{p} \sum_{k=0}^{m-1}\left(\begin{array}{c}
m \\
m-k-1
\end{array}\right) \frac{(-2)^{k}}{k !} \\
& \times \int_{0}^{\infty} e^{-y} y^{k+1} \sin z y d y, \quad z=m p .
\end{aligned}
$$

The last integral can be done explicitly and we get

$$
\begin{aligned}
\tilde{\gamma}_{m s}\left(p, p^{\prime}=0\right)= & 32 \pi(-1)^{m+1} \frac{m^{2}}{p} \sum_{k=0}^{m-1}\left(\begin{array}{c}
m \\
m-k-1
\end{array}\right) \frac{(-2)^{k}}{k !} \\
& \times \frac{(k+1) !}{\left(1+z^{2}\right)^{1+k / 2}} \sin [(k+2) \arctan z] .
\end{aligned}
$$

Let us call $\theta=\arctan z$, then $\cos \theta=1 / \sqrt{1+z^{2}}$ and $\sin \theta$ $=z / \sqrt{1+z^{2}}$. If we now take into account the trigonometric definition of the Chebyshev polynomials of the second kind $U_{n}(z)$ [which are a special case of the ultraspherical or Gegenbauer polynomials $\left.C_{n}^{(\alpha)}(z)\right]$ as

$$
U_{n}(\cos \theta)=\frac{\sin [(n+1) \theta]}{\sin \theta}=C_{n}^{(1)}(\cos \theta),
$$

we finally obtain

$$
\begin{aligned}
\tilde{\gamma}_{m s}\left(p, p^{\prime}=0\right)= & 32 \pi(-1)^{m+1} m ! m^{3} \sum_{k=0}^{m-1} \frac{(-2)^{k}}{(m-k-1) ! k !} \\
& \times \frac{1}{\left[1+(m p)^{2}\right]^{(k+3) / 2}} U_{k+1}\left(\frac{1}{\sqrt{1+(m p)^{2}}}\right) .
\end{aligned}
$$

In spite of the presence of the square roots, this expression is nothing but a quotient of polynomials in the variable $p^{2}$.

\section{APPENDIX B: THOMAS-FERMI RESULT FOR X-RAY SCATTERING FACTOR}

As discussed earlier by one of us [15]

$$
n_{T F}(r)=\frac{\sqrt{2}}{3 \pi^{2}}\left[\mu+\frac{Z e^{2}}{r}\right]^{3 / 2},
$$

where $\mu$ is the chemical potential. For $N$ electrons singly occupying $\mathcal{N}$ closed shells, with Eq. (3) relating them, one readily finds from $\int n_{T F}(r) d \mathbf{r}=N$ that

$$
\mu=-\frac{\sqrt{2}}{3 \pi^{2}} \frac{Z^{2}}{N^{2 / 3}} .
$$

The x-ray scattering factor corresponding to Eq. (B1) is given from Eq. (2) by

$$
f_{T F}(k)=\frac{\sqrt{2}}{3 \pi^{2}} \int_{0}^{R_{C}}\left[\mu+\frac{Z e^{2}}{r}\right]^{3 / 2} 4 \pi \frac{\sin k r}{k r} r^{2} d r,
$$

where $R_{c}$ is the classical radius defined from Eq. (B1) by

$$
R_{c}=-\frac{Z e^{2}}{\mu} .
$$

Putting $t=r / R_{c}$, Eq. (B3) then yields

$$
\begin{aligned}
f_{T F}(0)=N & =\frac{4 \sqrt{2}}{3 \pi}\left(Z e^{2} R_{c}\right)^{3 / 2} \int_{0}^{1}\left[\frac{1}{t}-1\right]^{3 / 2} t^{2} d t \\
& =\frac{\sqrt{2}}{12 \pi}\left(Z e^{2} R_{c}\right)^{3 / 2} .
\end{aligned}
$$

Indeed, the exact result can be obtained for any value of $k$ in terms of a generalized hypergeometric function as

$$
f_{T F}(k)=\frac{\sqrt{2}}{12 \pi}\left(Z e^{2} R_{c}\right)^{3 / 2}{ }_{2} F_{3}\left(\frac{3}{4}, \frac{5}{4} ; \frac{3}{2}, 2, \frac{5}{2} ;-\frac{\left(k R_{c}\right)^{2}}{4}\right),
$$

which is valid provided $R_{c}>0$. For small values of $k$, we have the approximate expression

$$
f_{T F}(k)=\frac{\sqrt{2}}{12 \pi}\left(Z e^{2} R_{c}\right)^{3 / 2}\left[1-\frac{\left(k R_{c}\right)^{2}}{32}+\frac{\left(k R_{c}\right)^{4}}{5120}-\cdots\right] .
$$

We plot $f_{T F}(k)$ in Eq. (B6) as a function of $k$ and also $R_{c}$ in Fig. 5. 


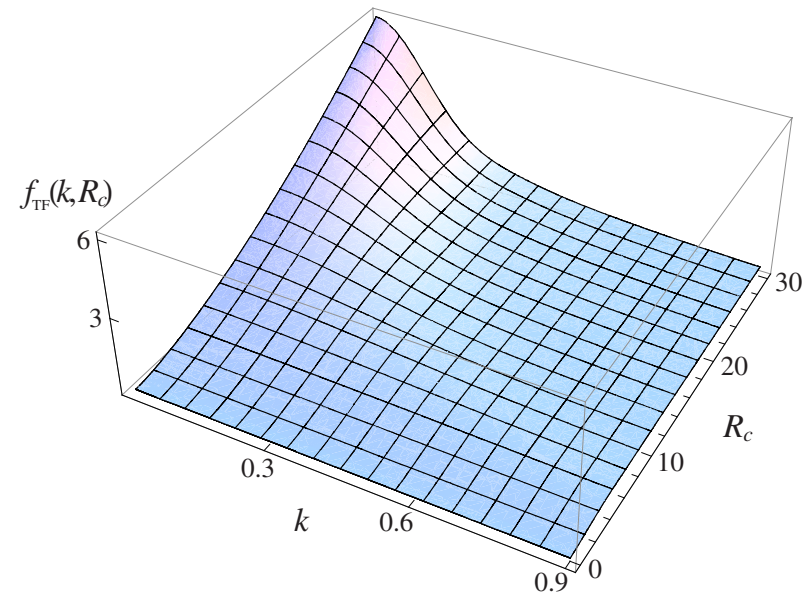

FIG. 5. (Color online) Plot of Thomas-Fermi approximation $f_{T F}(k)$ to x-ray scattering factor in Eq. (B6) as a function of $k$ and $R_{c}$. All the quantities are in atomic units.

For comparison with the exact quantal result

$$
f_{\mathcal{N}}(k)=\sum_{m=1}^{\mathcal{N}} f_{m}(k),
$$

the small $k$ expansion of $f_{\mathcal{N}}(k)$ follows as

$$
f_{\mathcal{N}}(k)=f_{\mathcal{N}}(0)+f_{2} k^{2}+\cdots .
$$

Turning to the (singular) case when $\mathcal{N}$ tends to infinity, and taking into account the integral

$$
\begin{aligned}
\int_{0}^{\infty} & \frac{1}{r^{3 / 2}} J_{3}(\alpha \sqrt{r}) \frac{\sin k r}{k r} 4 \pi r^{2} d r \\
= & \frac{8 \pi}{k \alpha}\left[\frac{16 k}{\alpha^{2}} \sin ^{2}\left(\frac{\alpha^{2}}{8 k}\right)-\sin \left(\frac{\alpha^{2}}{4 k}\right)\right],
\end{aligned}
$$

we can utilize the Heilmann-Lieb density $n_{\infty}(r)$ in their Eq. (2.4) to arrive at the result, using Eq. (2) once more:

$$
\begin{aligned}
f_{\infty}(k)= & \frac{2 \sqrt{2}}{\pi k} \int_{0}^{\infty} d x x e^{-x} \phi(x) \int_{0}^{\pi} d \theta w(x, \theta) \frac{1}{\alpha} \\
& \times\left[\frac{16 k}{\alpha^{2}} \sin ^{2}\left(\frac{\alpha^{2}}{8 k}\right)-\sin \left(\frac{\alpha^{2}}{4 k}\right)\right],
\end{aligned}
$$

where

$$
\alpha=2 \phi(x) \sqrt{2 w(x, \theta)} .
$$

Here $\phi(x)$ and $w(x, \theta)$ are given in [9] as

$$
\phi(x)=\sqrt{x /\left(1-e^{-x}\right)}
$$

and

$$
w(x, \theta)=1+e^{-x}-2 e^{-x / 2} \cos \theta .
$$

To conclude this appendix, we add some explicit results for small $m$ for the x-ray scattering factor $f_{m s}(k)$ introduced below Eq. (32) of the main text. If we call the relevant integral

$$
A_{m}(q)=\int_{0}^{\infty} e^{-t}\left[L_{m-1}^{(1)}(t)\right]^{2} t^{2} \frac{\sin q t}{q t} d t,
$$

we have the following results for the first five values of $m$ :

$$
\begin{gathered}
A_{1}(q)=\frac{2}{\left(1+q^{2}\right)^{2}}, \\
A_{2}(q)=\frac{8\left(1-3 q^{2}+2 q^{4}\right)}{\left(1+q^{2}\right)^{4}}, \\
A_{3}(q)=\frac{6\left(3-28 q^{2}+72 q^{4}-48 q^{6}+9 q^{8}\right)}{\left(1+q^{2}\right)^{6}}, \\
A_{4}(q)=\frac{32\left(1-19 q^{2}+109 q^{4}-215 q^{6}+166 q^{8}-46 q^{10}+4 q^{12}\right)}{\left(1+q^{2}\right)^{8}}, \\
A_{5}(q)=\frac{10\left(5-160 q^{2}+1626 q^{4}-6380 q^{6}+11190 q^{8}-9040 q^{10}+3330 q^{12}-500 q^{14}+25 q^{16}\right)}{\left(1+q^{2}\right)^{10}},
\end{gathered}
$$

thereby confirming for small $m$ the form $p_{2 m-2}\left(k^{2}\right) /\left(1+k^{2}\right)^{2 m}$ stated in the main text following Eq. (32). The x-ray scattering factor $f_{m s}(k)$ is then

$$
f_{m s}(k)=\frac{1}{2 m^{2}} A_{m}(k m / 2) .
$$


[1] M. Cohen, Adv. At. Mol. Phys. 25, 195 (1988).

[2] N. H. March, Phys. Rev. A 33, 88 (1986).

[3] T. Kato, Commun. Pure Appl. Math. 10, 151 (1957).

[4] M. S. Yusaf, G. P. Lawes, and N. H. March, Int. J. Quantum Chem. 17, 833 (1980).

[5] I. A. Howard, N. H. March, and V. E. Van Doren, Phys. Rev. A 63, 062501 (2001).

[6] A. K. Theophilou and N. H. March, Phys. Rev. A 34, 3630 (1986).

[7] M. Abramowitz and I. A. Stegun, Handbook of Mathematical
Functions (Dover, New York, 1972), Chap. 6.

[8] C. A. Coulson and N. H. March, Proc. Phys. Soc., London, Sect. A 63, 367 (1950)

[9] O. J. Heilmann and E. H. Lieb, Phys. Rev. A 52, 3628 (1995).

[10] R. M. May, Phys. Rev. 115, 254 (1959).

[11] V. Fock, Z. Phys. 98, 145 (1935).

[12] I. L. Cooper, Phys. Rev. A 50, 1040 (1994).

[13] S. M. Blinder, Phys. Rev. A 43, 13 (1991).

[14] B. R. Holstein, Am. J. Phys. 63, 710 (1995).

[15] N. H. March, J. Chem. Phys. 79, 3404 (1983). 